\title{
Studying Collaborative Object Positioning in Distributed Augmented Realities
}

\author{
Jens Müller, Simon Butscher, Stefan P. Feyer, and Harald Reiterer \\ HCI Group, University of Konstanz, Germany \\ \{jens.mueller,simon.butscher,stefan.feyer,harald.reiterer\}@uni-konstanz.de
}

\begin{abstract}
Augmented Reality (AR) displays have been suggested as shared-space technology to support remote collaboration, e.g., in design and building tasks. But with AR displays, the shared space typically consists of only the virtual work objects (e.g., design artifacts) while collaborators' interaction is grounded in their individual, physical environment. This can become problematic during activities that involve the positioning of virtual objects because the collaborators may require shared spatial references to coordinate their actions. In a lab experiment with 16 dyads, we studied how collaborators deal with that issue, and whether the provisioning of additive, virtual landmarks influences collaboration. As a result the landmarks improved user experience and decreased the reported temporal demand. In addition, we identified task-specific problem situations and provide implications for the design of distributed ARs to facilitate the collaborative positioning of virtual objects.
\end{abstract}

\section{Author Keywords}

Augmented Reality; remote collaboration; object positioning; virtual landmarks; user experience.

\section{INTRODUCTION}

Research has identified several application areas in which colocated collaboration can benefit from Augmented Reality (AR) technology, e.g., collaborative geometric modeling [14] and collaborative construction simulations [13] (see Lukosch et al. [18] for an overview). AR has also been suggested as a technology to create distributed augmented spaces to support building tasks such as those involved in collaborative design [31, 32] and molecular modelling [7]. Such activities require the collaborators to position virtual representations of the application-specific work objects (e.g., atoms for molecular modelling) in their shared work space. To coordinate their

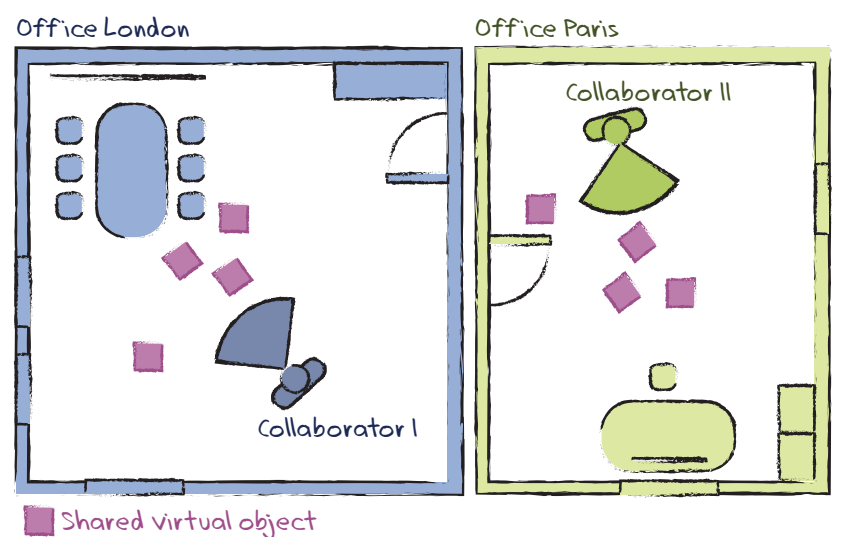

Figure 1. The potential problem with effective references in AR-based remote collaboration: To coordinate spatial actions, collaborator I and II can only rely on shared virtual objects because physical objects (such as office chairs) may either have different spatial relations to the virtual objects or do not exist in the other collaborators' environment.

actions, e.g., to collaboratively determine where to position a particular work object, collaborators must be able to generate effective references $[5,22]$. Chastine et al. [5] refer to this ability as "inter-referential awareness." But in distributed, collaborative ARs, only the shared virtual objects can serve as effective references because the physical objects are typically unique to each collaborator's physical environment (Figure 1). This may lead to problematic situations that make the coordination of actions difficult: For example, when the work space does not yet contain any shared virtual objects (and therefore does not provide effective references) an initial object positioning problem may occur. In this case it may become difficult to collaboratively determine where to position the first object. Previous work (e.g., [22]) has identified additive, virtual objects-so-called shared virtual landmarks (SVLs)-as effective references when collaborators have to guide each other's attention to virtual work objects in distributed ARs.

In this work we analyze how to support collaborative positioning of virtual work objects in distributed ARs focusing on following questions:

- Does the provisioning of SVLs support the positioning of virtual objects in distributed ARs?

- What possible problematic situations does object positioning entail and how do collaborators deal with them?

- What are the implications for the design of distributed ARs to facilitate activities that involve the positioning of objects? 


\section{RELATED WORK}

To provide a structured approach to the design of distributed ARs as shared-space technology, we first discuss critical design parameters of shared-space collaboration and their influence on collaboration. In the second part, we present studies on remote collaboration in $A R$ that provide further insights into how to support collaborative positioning of virtual objects in distributed ARs.

\section{Design Parameters of Shared-Space Collaboration}

Benford et al. [1] introduce a taxonomy consisting of the three dimensions Artificiality, Transportation, and Spatiality to classify shared space technologies. Additionally, the authors establish a link between these critical characteristics and their effect on user experience in collaborative situations. Their taxonomy offers several predictive aspects that enable the derivation of hypotheses. In the following we introduce the dimensions of the taxonomy and reflect on the implications that can be drawn with respect to collaborative object positioning in spatially distributed ARs.

Artificiality "concerns the degree to which the shared space is based on real-world information or is synthesized" [1]. With AR-based remote collaboration, the individual's space contains both information which is based on real-world information (the physical environment as provided by the see-through functionality) and the virtual work objects. According to this definition, AR technology can therefore be located somewhere in between the two extremes "Physical Reality" and "Virtual Reality," similar to the "virtuality continuum" as described by Milgram and Kishino [20].

Transportation "concerns the extent to which a group of participants and objects leave behind their local space and enter into some new remote space in order to meet with others, versus the extent to which they remain in their local space and the remote participants and objects are brought to them" [1]. As examples the authors refer to physical face-to-face collaboration where users remain in their physical context (no Transportation) and collaborative virtual environments where the users meet in virtual reality and their physical context becomes less significant (high degree of Transportation). At this point Benford et al. [1] establish a link between Artificiality and Transportation: The more Artificiality a medium can provide, the more insignificant the physical context can become and the more it can support the sense of being transported.

Spatiality "concerns the degree to which the shared space exhibits key spatial properties such as containment, topology, movement, and a shared frame of reference" [1]. The provisioning of these properties increases the "reciprocity of perspective" [1]. Thus, increased Spatiality can support a collaborator's "ability to refer to a set of objects, and for that reference to be understood by others" [5], which Chastine et al. [7] refer to as "inter-referential awareness". Collaborative Virtual Realities, for example, can provide a shared coordinate system and a shared frame of reference. This allows the collaborators to infer each other's perspective on the shared information space and to coordinate their spatial actions accordingly. Benford et al. [1] note that the degree of required Spatiality depends heavily on the task.

\section{Studies Related to the Design Parameters}

The effect of Spatiality has been well researched in terms of shared visual context (e.g., [5, 6, 8, 9, 10, 11, 23]). For example, Gergle et al. [10] studied how different levels of shared visual information influences collaboration behaviour and performance. Collaborators had to jointly solve a puzzle with one collaborator being the remote instructor and a second collaborator being the executing worker. Their study revealed that shared visual information makes communication more efficient and improves task performance. Similarly, Gauglitz et al. [9] presented an AR prototype which supports an "augmented shared visual space for live mobile remote collaboration on physical tasks" [9]. A key feature of their prototype referred to virtual landmarks that served as "worldstabilized annotations" [9]. Chastine et al. [5, 6] studied how collaborators perform a building task under several conditions (co-located and remote, AR and VR). They highlight the "bizarre challenges" [5] of AR as shared spaces: "because AR space is comprised of both the physical and virtual objects that surround the user, the addition of each new remote participant to the shared space brings with it the environment that surrounds them, creating a composite of overlapping environments" [5]. In their study they found that collaborators made extensive use of deictic speech. As a consequence they suggest that collaborators "need reference points embedded within the environment to more easily generate references" [5]. To reduce orientation disparities and other asymmetries during video-based remote collaboration, O'Hara et al. [23] suggest the provisioning of "shared spatial geometries" such as horizontal and vertical multipoint surfaces.

The effect of different degrees of Artificiality and Transportation has been studied primarily in terms of mixed collaboration between AR, VR, and physical environments ([2, 12, 27, 28]). Billinghurst [2] introduced the MagicBook prototype, a transitional interfaces that allow the user to switch between interface types (e.g., AR and VR), and from remote to co-located collaboration. Grasset et al. [12] present a prototype where users can interact from different spaces-forming a mixedspace environment-which differ in terms of their immersion and perspective. In addition, they introduce the "transitional collaborative model," a conceptual model that illustrates the different collaboration types of transitional interfaces. Similarly, Stafford [28] investigates how visual cues influence collaborative navigation tasks in a mixed-space environment. As a result, visually cued, collaborative navigation techniques were more efficient than an audio-only technique.

Several studies have established the positive effect of SVLs on AR-based collaboration. Müller et al. [21] studied both object identification (dyads had to guide each other's attention to virtual work objects in their AR) and object positioning (dyads had to position virtual objects in their AR) in a co-located setting. A follow-up study [22] investigated how the provisioning of SVLs influences the process of object identification when collaborators work in distributed ARs. In this study they analyzed possible conflict situations that are specific to both the task (object identification) and the study condition (SVLs provided vs. no SVLs provided). In addition, they analyzed Transportation and found that collaborators reported and in- 
creased sense of being transported to a new place when SVLs provided. But participants did not report an increased sense of being together in the same new place.

\section{Relationship to Previous Work}

Previous work has investigated the role of SVLs in different spatial settings (co-located and distributed) and for different spatial tasks (e.g., object identification and object positioning), in terms of co-located object positioning (e.g., [21]) and distributed object identification (e.g., [22]). This study extends these findings by investigating how SVLs shape the process of collaborative object positioning in distributed ARs. Furthermore, it analyzes task-specific problem situations and provides implications for the design of distributed ARs to facilitate activities that involve the positioning of objects. Moreover, the current study provides a theoretical foundation and discussion of results based on the classification of shared-space technologies by Benford et al. [1].

\section{FORMULATION OF HYPOTHESES}

For the collaborative positioning of virtual objects in distributed ARs, we consider Spatiality [1], a key dimension, and SVLs, a key spatial property (e.g., [5, 9, 22]). We therefore hypothesize that:

$\mathrm{H}_{1}$ : The provisioning of SVLs positively influences collaborative object positioning in distributed ARs.

Furthermore, SVLs increase the degree to which the shared space is synthesized and thus increases Artificiality [1]. Based on the relation between Artificiality and Transportation [1, 2, 22], the physical environment becomes less significant for the collaborators which causes an increased sense of Transportation. An increased sense of Transportation caused by SVLs has been shown by Müller et al. [22] for collaborative object identification in distributed ARs. Participants, however, did not report an increased sense of being together in the same place. We assume that collaborative object positioning is a more complex task than object identification: object identification merely requires the collaborators to exchange spatial information about an already existing object in the shared space, whereas object positioning requires them to exchange spatial information about the assumed and not yet defined positions in mid-air. As a consequence, during object positioning the SVLs may play an even more important role in the collaborators' perception. We therefore hypothesize that:

$\mathrm{H}_{2}$ : The provisioning of SVLs leads to an increased sense of being together in the same place.

\section{STUDY}

We conducted a controlled lab experiment using a counterbalanced within-subjects design with the provisioning of SVLs being the independent variable. The baseline condition refers to the condition when no SVLs were provided.

\section{Study Environment and Apparatus}

Two rooms of our research facility served as physically separate AR work spaces for our participants. Each room was equipped with a video camera providing a long shot view of the work area. For AR displays we used Google's Project
Tango tablets [29]. To enable communication between collaborators and the recording of communication we installed Teamspeak 3 on the tablets [30]. We used a dedicated server to synchronize tablet positions, interaction events, task states, and interaction events and stored them in a log file.

\section{Study Task}

Collaborative object positioning assumes a common notion regarding the objects' locations. To establish such a common notion, we used a collaborative AR memory game as suggested by Müller et al. [21, 22]: We distributed a set of 20 white, virtual cubes with an edge length of $15 \mathrm{~cm}$ within a physical $3 \mathrm{D}$ volume of approx. $4 \mathrm{~m} \times 3 \mathrm{~m} \times 2 \mathrm{~m}$ in the collaborators' environment (Figure 2). We used 10 different symbols from the Wingdings font and assigned each symbol to two cubes, forming 10 pairs of cubes. Each turn, collaborators had to find cubes with the same symbol. Collaborators could select any cube by touching its $3 \mathrm{D}$ representation on the tablet screen. Once a cube was selected, it revealed its symbol. Collaborators were able to communicate via the tables to coordinate their actions. Each collaborator was allowed to select only one cube per turn. If the two selected cubes unveiled the same symbol, they were removed from the shared work space, otherwise they turned white again by the system. Collaborators were tasked with finding all pairs in as few turns as possible. This part was has been studied in previous work [22] and was therefore not considered in the analysis.

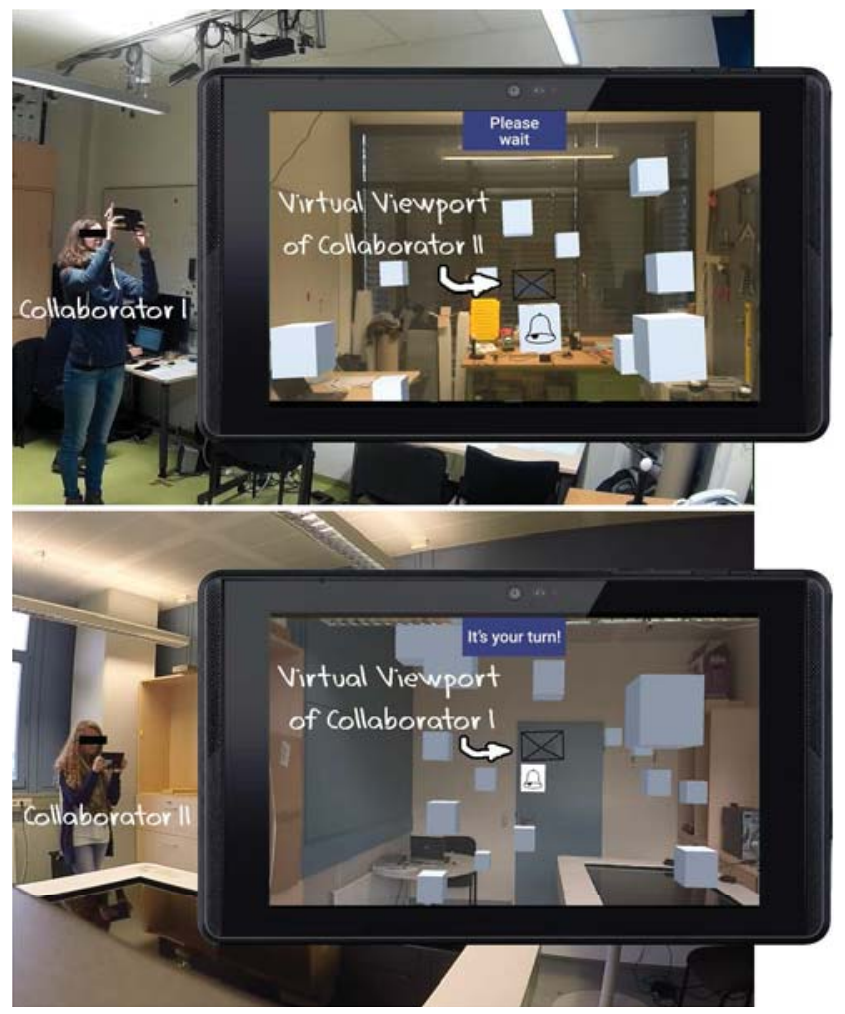

Figure 2. Collaborative AR memory game a: Collaborator I and II can see each other's tablet on their own tablet. In this example collaborator II has has uncovered a cube showing the sign of a bell. Collaborator I now needs to find and uncover the cube that contains the second bell. 
In the reconstruction part, participants were tasked with positioning the symbol cubes according to their positions in the previous AR memory game. Each collaborator was allowed to position only one cube of each of the ten pairs by pressing its button representation on the border of the screen (Figure 3). In front of each collaborator's tablet there was a proxy cube providing a preview of where the cube would appear in 3D space. Collaborators saw each other's tablets and proxy cubes. Object positioning was not designed as a turn-based task. A positioned cube could be removed by any of the collaborators by touching its $3 \mathrm{D}$ representation on the tablet screen. That cube could then be positioned elsewhere.

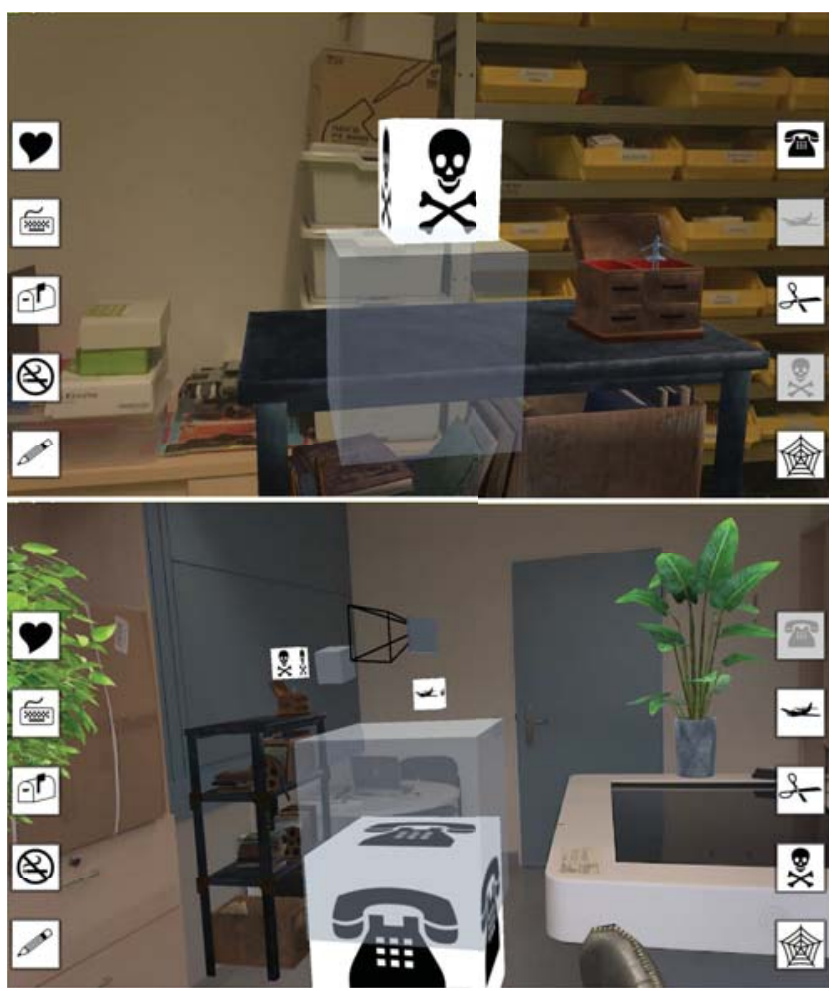

Figure 3. Tablet views of collaborator I (top) and collaborator II (bottom, observing collaborator I) during the object positioning task. In the example collaborator I has just positioned the skull cube and collaborator II the telephone cube.

\section{Dependent Variables, Measures, and Analysis}

We use a mixed-methods approach focusing on four aspects:

- User Behavior was analyzed in terms of collaborators' verbal Spatial Expressions and their Strategies to accomplish the task. All interactions were video recorded with a long shot camera in each room. In addition, audio was captured on the tablet using TeamSpeak 3 and the recording plugin [30]. From 16 synchronized video files we exported those parts that contained the object positioning task and proceeded with them. We took half of the video sessions and analyzed them for Spatial Expressions following an open coding approach. We then created categories based on the transcribed expressions. For category names we referred to naming conventions proposed by Müller et al. [22]. We defined an additional label for potentially interesting Strategies. Afterwards we analyzed the video from all sessions using the categories as a coding scheme. All occurrences that referred to potentially interesting Strategies were then re-examined qualitatively.

- User Experience was analyzed in terms of Preferences and Transportation. Users' Preferences were examined within a concluding, semi-structured interview including the rating of study conditions on a Likert scale and several open-ended questions about the perceived helpfulness of virtual and physical elements. The interview was conducted with both participants in the same room but questions were answered individually. Transportation was assessed using the subdimension "Parasocial Interaction" of the Temple Presence Inventory (TPI) questionnaire [17].

- Subjective User Workload was assessed using the NASA TLX questionnaire [24].

- Team Performance was measured in terms of Positioning Error. For the calculation of Positioning Error we considered the two correct coordinates of a pair of cubes and the two coordinates of the positioned cubes and determined the two combinations that yielded the smallest error. Assessment of both variables is based on the interaction log file which was generated during each session and stored on a dedicated study server.

\section{Study Procedure}

Participants were welcomed and introduced to the study. Then each participant was brought to their individual room. Afterwards, each participant was asked to fill out a demographic questionnaire. Upon completion, participants familiarized themselves with the tablets and the task. SVLs were not provided during familiarization and a training set of cubes (consisting of training coordinates and numbers from one to ten instead of symbols). Participants then started with the 3D memory game (approx. 10 minutes) in their (randomly assigned) study condition (either baseline or SVL). Then, participants were asked to answer the NASA TLX and the user experience questionnaire containing the Transportation items. Afterwards, participants continued with the object positioning task in the same condition (approx. 8 minutes). Then, participants were again asked to fill out the NASA TLX questionnaire and the user experience questionnaire. Both tasks and assessments were repeated in the respective other study condition using a different set of coordinates and symbols for the cubes to avoid carry-over effects. After completion of the second iteration, one participant was brought to the room of the other one for the concluding, semi-structured interview. Each session took approx. 50 minutes.

\section{Participants}

We recruited 32 participants (12 male, 20 female) between 19 to 29 years of age, forming 16 dyads. 29 participants were university students and 3 were employees.

\section{Findings}

Results referring to the baseline condition (no SVLs) are marked with subscript ${ }_{\mathrm{B}}$ and results referring to the landmarks 
condition with subscript SVL. To analyze for statistical significance we used the non-parametric Wilcoxon signed-rank test and refer to the median $(M d n)$ if the normal distribution was not given and for ordinal data. Otherwise, Student's $t$-test was used and mean values $(M)$ are reported.

\section{User Behavior}

For Spatial Expressions video coding yielded a set of 7 categories:

- Deictic Speech (expressions that "cannot be fully understood by speech alone," [16] e.g., "here," "there")

- Other Cube (e.g., "above the cube with the bell symbol")

- Person (e.g., "in front of you")

- Maneuvering Command (e.g., “ up, up, up, up, left”)

- Room (e.g., "on the floor")

- Physical Object (e.g., "near the window")

- Shared Virtual Landmark (e.g., "above the armchair")

On average, dyads made 49.50 spatial expressions in the baseline condition $\left(S D_{\mathrm{B}}=16.37\right)$ and 49.37 spatial expressions in the landmarks condition $\left(S D_{\mathrm{SVL}}=14.89\right)$ (Figure 4$)$. A $t$-test revealed that in the landmarks condition, collaborators made significantly less use of deictic speech, referred to other virtual cubes less frequently, and used the landmarks more frequently (Deictic Expression: $M_{\mathrm{B}}=30.37, S D_{\mathrm{B}}=10.85, M_{\mathrm{SVL}}=24.18$, $S D_{\mathrm{SVL}}=8.78, t(15)=2.88, p=0.01$; Other Cube: $M_{\mathrm{B}}=9.12$, $S D_{\mathrm{B}}=5.30, M_{\mathrm{SVL}}=5.56, S D_{\mathrm{SVL}}=3.36, t(15)=2.38, p=0.03$; Shared Virtual Landmark: $M_{\mathrm{B}}=0.18, S D_{\mathrm{B}}=0.54, M_{\mathrm{SVL}}=11.50$, $\left.S D_{\mathrm{SVL}}=5.13, t(15)=-8.90, p<0.01\right)$.

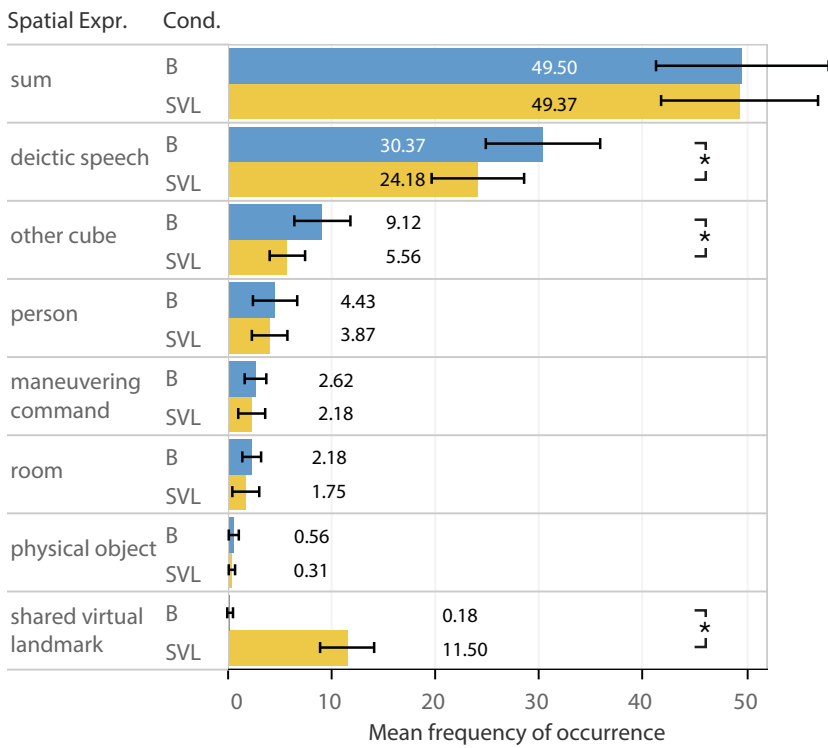

Figure 4. Categories of spatial expressions and their mean frequency of occurrence per study session.

With respect to Strategies, we observed that collaborators sometimes positioned one of their remaining cubes without consultation of their partner. But as soon as they collaboratively positioned a cube, there was frequently a collaboration asymmetry: one collaborator took the role of the helper who gave the worker maneuvering commands, such as "up-upup-left-left," based upon which the worker performed "small, precise movements" [3]. This remote-control behaviour occurred in 15 groups in the baseline condition, and in 10 groups in the landmarks condition. Another frequent pattern refers to the participants' perspective and movement: Regardless of the study condition, most participants $\left(\mathrm{N}_{\mathrm{B}}=28, \mathrm{~N}_{\mathrm{SVL}}=23\right)$ chose a specific position within their room from which they initiated positioning. After they had positioned a cube they would typically return to that position.

\section{User Experience}

Considering Preference, all participants favoured the landmarks condition. Reasons for which they indicated that are: that the landmarks made the cubes easier to memorize $(\mathrm{N}=2)$, that the landmarks provided better orientation $(\mathrm{N}=4)$, that the landmarks provided better referencing possibilities $(\mathrm{N}=12)$, that the landmarks improved communication $(\mathrm{N}=4)$, and that they were able to better share their orientation by using the landmarks $(\mathrm{N}=3)$. In the interview, we also asked participants independently to rate how helpful the physical environment was on a scale from 1 to 10. Participants answers ranged from 1 to 10 with a median of 6.00 . For ratings that were in favor of the physical environment, participants gave following explanations: The physical environment served as an aid to general orientation $(\mathrm{N}=5)$, that it provides an orientation frame $(\mathrm{N}=1)$, and that they used their individual physical environment to memorize object locations $(\mathrm{N}=5)$.

Several participants mentioned the physical environment in terms of interference $(\mathrm{N}=10)$, e.g., "it did not provide any useful reference," "you automatically generate a second room, based on the physical one," "concentration on one's own environment is obstructive," "I had to imagine they [the real objects] weren't there," and "I had to think the real objects away"). Another 2 participants mentioned that the physical environment did not influence them at all ("[the task] could have taken place in empty space as well," "I could have played in the dark as well"). Furthermore, 14 participants commented that the physical environment became less significant when additive virtual objects were provided.

As a final question, we asked participants to think of any features that might have helped them in solving the task. There were 2 main themes in the answers. The first theme referred to the room itself: participants from 6 groups mentioned that a division of the work space such as virtual "reference lines," "layers," or a "virtual grid" would have been helpful. Participants from 4 groups mentioned the harmonization of work spaces as potential improvement, e.g., through a "new virtual, but identical room" or by providing virtual "walls" in the physical room. The second theme referred to desired functionality. Participants from 5 groups wished for pointing functionality, e.g., to make others aware of a particular object or position or as tool to "measure distances" and position the cubes "as the proxy cube only allowed for the positioning directly in front of one's self."

Considering Transportation, a Wilcoxon signed-rank test revealed that participants' rankings were significantly higher when the landmarks were provided (Figure 5) for the item 
LEFTPLACE ("How much did it seem as if you and the people you saw/heard both left the places where you were and went to a new place?") and the item TOGETHER ("How much did it seem as if you and the people you saw/heard were together in the same place?"). In addition, in the landmarks condition participants ranked the item CONTROL significantly higher when the landmarks were provided ("Seeing and hearing a person through a medium constitutes an interaction with him or her. How much control over the interaction with the person or people you saw/heard did you feel you had?"). (LEFTPLACE: $M d n_{\mathrm{B}}=3, M d n_{\mathrm{SVL}}=5, Z=-2.31$, $p=0.02$; TOGETHER: $M d n_{\mathrm{B}}=4.5, M d n_{\mathrm{SVL}}=5, Z=-3.26$, $p<0.01$; CONTROL: $M d n_{\mathrm{B}}=5, M d n_{\mathrm{SVL}}=5, Z=-2.28, p=0.02$ )

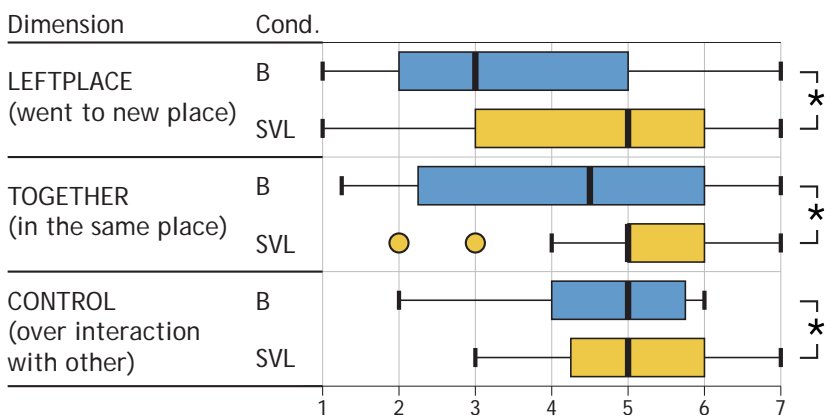

Figure 5. Items of the sub-dimensions of the Temple Presence Inventory that yielded significant differences.

\section{User Task Load}

A Wilcoxon signed-rank test did not yield significant difference in the overall task load between the two conditions. Analysis of the subscales revealed a significantly lower temporal demand in the landmarks condition than in the baseline condition ("How much time pressure did you feel due to the pace at which the tasks or task elements occurred? Was the pace slow or rapid?" $M d n_{\mathrm{B}}=45, M d n_{\mathrm{SVL}}=35, Z=-1.98, p=0.04$ ) (Figure 6)

\begin{tabular}{ll|llllllllllll} 
Subscale Cond. \\
$\begin{array}{l}\text { temporal } \\
\text { demand }\end{array}$
\end{tabular}

Figure 6. Median score of the NASA-TLX subscale "temporal demand."

\section{Team Performance}

A $t$-test did not reveal a statistical difference in Positioning Error (In meter: $M_{\mathrm{B}}=25.4 \mathrm{~m}, S D_{\mathrm{B}}=5.0, M_{\mathrm{SVL}}=26.5 \mathrm{~m}$, $\left.S D_{\mathrm{SVL}}=3.6, t(15)=-0.56, p=0.58\right)$.

\section{DISCUSSION}

The discussion of results and associate implications is structured according to the initial research questions. In the hypothesis-driven part we addressed the following question:

Does the provisioning of SVLs support the positioning of virtual objects in distributed ARs?

Resonating with prior studies (e.g, [21, 22]), the provisioning of SVLs positively influenced collaboration: SVLs replaced ambiguous deictic expressions extensively, lead to an improved reported user experience, and participants preferred the condition in which SVLs were provided. Considering team performance, however, the provisioning of virtual landmarks did not decrease the average positioning error, although landmarks are known to support spatial memory [25]. One explanation could be that we did not provide depth cues such as lighting and shadows, which might have made it difficult for the participants to judge distances. Moreover, the tablets did not provide stereoscopic images and thus did not optimally support depth perception. Thus, referring to $\mathrm{H}_{1}$, as in related studies (e.g, [21, 22]), the provisioning of SVLs positively influenced user experience, but did not improve team performance.

In terms of Transportation, the provisioning of SVLs increased participants' sense of being transported in two ways: participants reported an increased sense of being transported to a new place but also reported an increased sense of being there together with their collaborator (unlike in the study by Müller et al. [22] who studied object identification in a distributed $\mathrm{AR})$. One explanation could be the rationale behind $\mathrm{H}_{2}$, that, in contrast to the identification of objects, the positioning of objects is a more complex task within which collaborators benefit more from the presence of SVLs. This is supported by the finding that participants also reported an increased control over the interaction with their collaborator when SVLs were provided. An alternative explanation could be that collaborators saw each other's proxy cubes. This visual enhancement of the collaborators may have increased their awareness for each other's actions and may have caused both an increased sense of transportation and control. According to participants' responses in the interview, however, not all participants found it easy to leave their local environment behind. Some reported that they had to consciously differentiate their physical environment from the shared landmarks. This suggests that there are inter-personal differences in terms of how strongly virtual objects dominate individuals' experiences and to what extend individuals perceive "orientation disparities" [23]. Thus, referring to $\mathrm{H}_{2}$, SVLs generally increased Transportation in the sense that collaborators reported an increased level of being transported to the same new place. But the provisioning on SVLs could also become problematic for some participants who had to actively differentiate them from the physical environment.

In the exploratory part of the study, we addressed the following questions:

What possible problematic situations does object positioning entail and how do collaborators deal with them?

What are the implications for the design of distributed ARs to facilitate the positioning of virtual objects?

It became apparent that ambiguity problems (collaborators refer to individual, physical objects to communicate spatial information about virtual objects to be positioned) occurred infrequently in any of the conditions. Furthermore, an initial object positioning problem did not occur, which can be explained by the fact that the collaborators were allowed to position cubes individually. Thereby collaborators could create 
their own initial landmarks using the work objects (represented by the cubes). Besides, collaborators could see each other's proxy cube which served as dynamic landmarks based upon which they could collaboratively positioned the initial cube (e.g., via remote-maneuvering). But participants reported a cognitive ambiguity problem that occurred before they made any spatial expression. In this situation participants first had to be clear on whether or not an object is suitable as a reference object prior to initiation of coordination processes with their partner. According to participants' experiences, physical objects were useful for individual orientation in their individual AR, but only the shared virtual landmarks were suitable for coordinating spatial actions.

Considering users' strategies, we found that most participants chose a certain perspective throughout a study condition and would only leave that point in order to position a cube. This resonates with some of the participants' statements that they wished to have a positioning tool/pointer that would allow them to stay in place and position cubes from a distance.

\section{IMPLICATIONS}

Referring to the cognitive ambiguity problem where participants had to decide whether or not an object is suitable as a reference object (where virtual objects were suitable and physical object were not), virtual landmarks should be designed in such a way that the viewer perceives them as virtual ones (see Table 1 for a summary of design suggestions).

Furthermore, some participants wished to have a positioning tool/pointer that would allow them to stay in place and position cubes from a distance. A simple pointer, however, might not be suitable as it does not provide the means to position virtual objects in mid-air, nor would it support collaboration. We suggest the concept of collaborative pointing that would make it possible to position virtual object in any position within a $3 \mathrm{D}$ volume (Figure 7). The concept requires at least two virtual pointers which can cast a virtual ray into the $3 \mathrm{D}$ volume. Virtual objects can be positioned at the intersection point of the two rays. As with our implementation of the proxy cube in front of each collaborator, a virtual proxy cube appears at

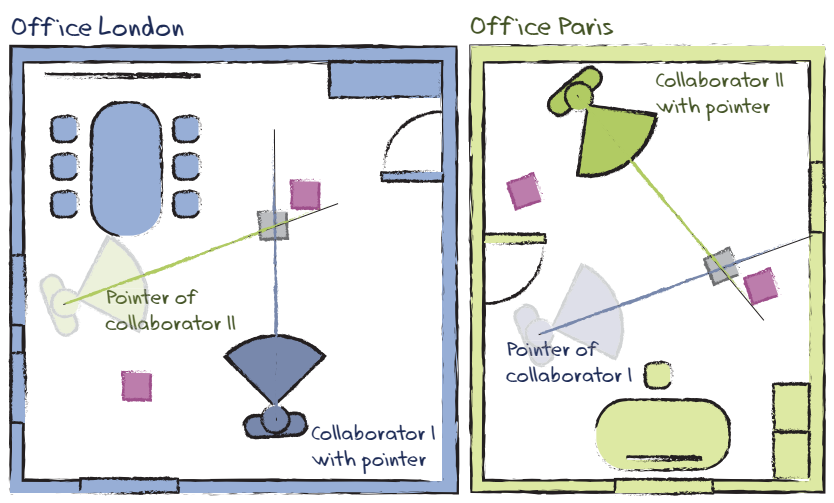

Shared, virtual object

Point of intersection, shows proxy of object to be positioned

Figure 7. Collaborative 3D Pointing: A virtual object can be positioned from a distance where the two pointing rays interfere.

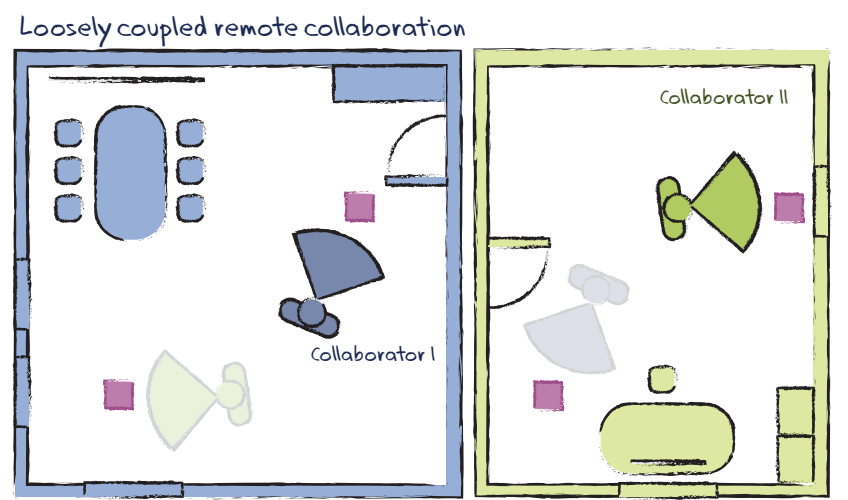

Tightly coupled remote collaboration

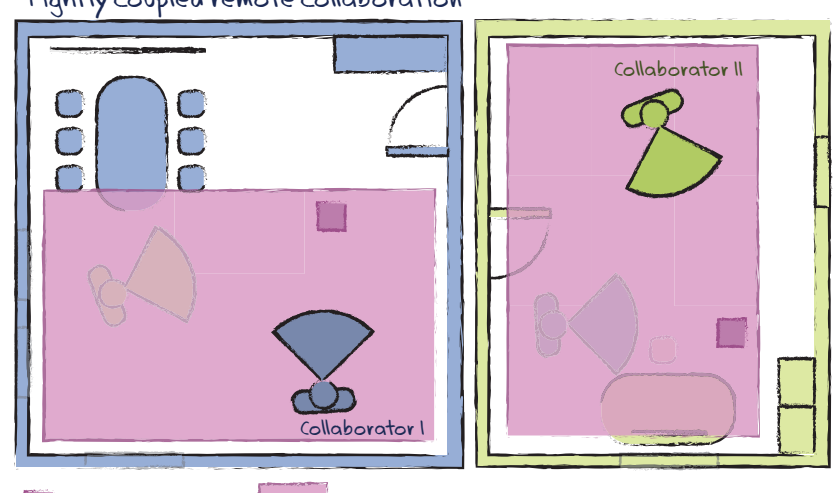

Virtual object Virtual room (display in VR mode)

Figure 8. Transitional devices to support remote collaboration. Top: If collaborators work loosely-coupled, their devices show their physical environment. Bottom: During tightly-coupled collaboration, e.g., when they focus on the same virtual object, their interfaces turn into VR mode, replacing their individual, physical environments with a shared, synthesized one.

the intersection point. As soon as the collaborators commit the current position of the proxy cube, the virtual cube is instantiated at that position.

Further implications on how to support remote-collaborative object positioning can be drawn from our observations in conjunction with participants' feedback. During the task, collaborators went trough phases where they positioned the cubes individually and phases of tightly-coupled collaboration (e.g., via remote-maneuvering). In addition, participants reported that the physical environment was helpful for their individual cognition, but became unhelpful or even disturbing in collaborative phases (cognitive ambiguity problem). Thus, tightly-coupled collaboration requires more Artificiality to allow the collaborators to leave behind the local and physical environment and to move towards virtual reality which "combines the remote and synthetic" [1]. To make the physical environment less significant, Artificiality could be increased by replacing collaborators' physical, visual context with a synthesized one such as the MagicBook interface [2]. The degree of Artificiality in the resulting transitional interface (Figure 8 ) could be controlled implicitly by the system. This would require the system to identify the current coupling style. Identification of coupling styles could be achieved by means 
of the collaborators' current communication behaviour (e.g., ongoing conversational turns indicate close collaboration). Additionally, the system could infer the current coupling style from the spatial relations (such as F-Formations [15]) between the collaborators as suggested in the proxemic interaction framework [19] (e.g., collaborators facing towards each other indicate close collaboration). Alternatively, the system could offer explicit control over the degree of Artificiality via a user control element.

Additional suggestions can be made based on participants' feedback. Several participants suggested "discretizing" the work spaces in terms of layers, grids, or frames. Adding such elements would increase Artificiality and Spatiality and could possibly increase Transportation and unify physically distributed work environments. Further interesting comments refer to desired, additional features. Several participants suggested providing a pointing device (such as suggested by Chastine et al. [4]) to better guide the other collaborator's attention.

Provide virtual landmarks that look artificial to make them distinguishable from individual, physical objects.

Provide a positioning tool that supports the collaborative positioning of virtual objects from a distance.

Provide a transitional interface to address collaborators' needs during loosely-coupled and tightly-coupled collaborative phases.

Discretize the shared workspace to increase Spatiality and to support the coordination of actions.

Table 1. Suggestions to support the collaborative positioning of virtual objects in distributed ARs

\section{LIMITATIONS AND FUTURE WORK}

Limitations of our study refer to the generalizability of results. In our study, participants used a certain display technology (AR tablets) to solve a certain type of task (object positioning). A different display technology may have yielded different results due to their individual ergonomic and perceptional qualities [26]. For example, the used AR tablets can only provide a limited AR experience due to their limited field of view into the AR space, whereas head-mounted displays can also augment peripheral vision. This assumedly influences critical aspects of remote collaboration such Transporation [1]. Therefore, the effect of AR display technology on collaborative work should be subject to future research.

Considering the type of task, this study focuses on the collaborative positioning of virtual work objects that do not semantically relate to the physical environment. This is an important differentiation, because in such scenarios, the physical environment merely serves as a containment of the virtual work objects. Such scenarios refer, for example, to distributed design [31], modelling [5], and planning [1] tasks.

In addition, different types of tasks involve different requirements $[1,10]$. Some remote-collaborative tasks, for example, require a high degree of Spatiality and should therefore possibly be carried out in collaborative virtual environments. Thus, further analysis on the special requirements (e.g., in terms of Transportation and Spatiality) in relation to different types of tasks is needed.

\section{CONCLUSION}

In a study with 16 dyads, we investigated whether shared virtual landmarks (SVLs) positively influence collaboration. Results partly resonate with the findings from previous research on the related task of object identification: The provisioning of SVLs improved user experience and decreased the use of deictic expressions but did not increase team performance. However, for object positioning the provisioning of SVLs also increased the collaborators' sensation of being transported to another, shared place. In addition, we identified several problem scenarios that are specific to object positioning in distributed ARs. Based on the findings of a mixed-methods approach we provide four design suggestions on how to facilitate activities that involve the positioning of virtual objects in distributed ARs.

\section{ACKNOWLEDGEMENTS}

The work was supported by SmartAct. We also thank Matthias Kraus and Matthias Miller for their hands-on support.

\section{REFERENCES}

1. Steve Benford, Chris Greenhalgh, Gail Reynard, Chris Brown, and Boriana Koleva. 1998. Understanding and Constructing Shared Spaces with Mixed-reality Boundaries. ACM Transactions on Computer-Human Interaction (TOCHI) 5, 3 (1998), 185-223. DOI : http://dx.doi.org/10.1145/292834.292836

2. Mark Billinghurst, Hirokazu Kato, and Ivan Poupyrev. 2001. MagicBook: Transitioning Between Reality and Virtuality. In Ext. Abstracts CHI 2001. ACM Press, 25-26. DOI : http://dx . doi .org/10.1145/634067.634087

3. Doug A. Bowman, Ernst Kruijff, Joseph J. LaViola, and Ivan Poupyrev. 2004. 3D User Interfaces: Theory and Practice. Addison Wesley Longman Publishing Co., Inc., Redwood City, CA, USA.

4. Jeffrey W. Chastine, Kristine Nagel, Ying Zhu, and Mary Hudachek-Buswell. 2008. Studies on the Effectiveness of Virtual Pointers in Collaborative Augmented Reality. In Proc. 3DUI 2008. IEEE, 117-124. DOI: http://dx. doi .org/10.1109/3DUI. 2008.4476601

5. Jeffrey W. Chastine, Kristine Nagel, Ying Zhu, and Luca Yearsovich. 2007. Understanding the Design Space of Referencing in Collaborative Augmented Reality Environments. In Proc. GI 2007. ACM Press, 207-214. DOI : http://dx. doi .org/10.1145/1268517.1268552

6. Jeffrey W. Chastine and Ying Zhu. 2008. The Cost of Supporting References in Collaborative Augmented Reality. In Proc. GI 2008. ACM Press, 275-282.

7. Jeffrey W. Chastine, Ying Zhu, and Jon A. Preston. 2006. A Framework for Inter-referential Awareness in 
Collaborative Environments. In Proc. CollaborateCom 2006. IEEE, 1-5. DOI :

http://dx.doi.org/10.1109/COLCOM.2006.361859

8. Susan R. Fussell, Robert E. Kraut, and Jane Siegel. 2000. Coordination of Communication: Effects of Shared Visual Context on Collaborative Work. In Proc. CSCW 2000. ACM Press, 21-30. DOI : http://dx.doi.org/10.1145/358916.358947

9. Steffen Gauglitz, Benjamin Nuernberger, Matthew Turk, and Tobias Höllerer. 2014. World-stabilized Annotations and Virtual Scene Navigation for Remote Collaboration. In Proc. UIST 2014. ACM Press, 449-459. DOI : http://dx.doi.org/10.1145/2642918.2647372

10. Darren Gergle, Robert E. Kraut, and Susan R. Fussell. 2004. Language Efficiency and Visual Technology: Minimizing Collaborative Effort with Visual Information. Journal of Language and Social Psychology 23, 4 (2004), 491-517. DOI:

http://dx.doi.org/10.1177/0261927X04269589

11. Darren Gergle, Robert E. Kraut, and Susan R. Fussell. 2013. Using Visual Information for Grounding and Awareness in Collaborative Tasks. Human-Computer Interaction 28, 1 (2013), 1-39. DOI : http://dx.doi.org/10.1080/07370024.2012.678246

12. Raphael Grasset, Philip Lamb, and Mark Billinghurst. 2005. Evaluation of Mixed-Space Collaboration. In Proc. ISMAR 2005. IEEE, 90-99. DOI : http://dx. doi.org/10.1109/ISMAR.2005. 30

13. Amin Hammad. 2009. Distributed Augmented Reality For Visualising Collaborative Construction Tasks. Springer Netherlands, Dordrecht, Netherlands, 171-183. DOI : http://dx. doi.org/10.1007/978-1-4020-9088-2_11

14. Hannes Kaufmann, Dieter Schmalstieg, and Michael Wagner. 2000. Construct3D: A Virtual Reality Application for Mathematics and Geometry Education. Education and Information Technologies 5, 4 (2000), 263-276. DOI :

http://dx.doi.org/10.1023/A: 1012049406877

15. Adam Kendon. 2009. Spacing and Orientation in Co-present Interaction. In Proc. COST 2009. Springer-Verlag, 1-15. DOI : http://dx.doi .org/10.1007/978-3-642-12397-9_1

16. Kiyoshi Kiyokawa, Mark Billinghurst, S. E. Hayes, A. Gupta, Y. Sannohe, and Hirokazu Kato. 2002. Communication Behaviors of Co-Located Users in Collaborative AR Interfaces. In Proceedings of the 1st International Symposium on Mixed and Augmented Reality (ISMAR '02). IEEE Computer Society, Washington, DC, USA, 139-148. DOI : http://dx.doi.org/10.1109/ISMAR.2002.1115083

17. Matthew Lombard, Theresa. B. Ditton, and Lisa Weinstein. 2009. Measuring Presence: The Temple Presence Inventory. In Proc. Presence 2009. ISPR.
18. Stephan Lukosch, Mark Billinghurst, Leila Alem, and Kiyoshi Kiyokawa. 2015. Collaboration in Augmented Reality. Computer Supported Coop Work (CSCW) 24, 6 (2015), 515-525. DOI : http://dx. doi .org/10.1007/s10606-015-9239-0

19. Nicolai Marquardt, Robert Diaz-Marino, Sebastian Boring, and Saul Greenberg. 2011. The Proximity Toolkit: Prototyping Proxemic Interactions in Ubiquitous Computing Ecologies. In Proc. UIST 2011. ACM Press, 315-326. DOI :

http://dx.doi.org/10.1145/2047196.2047238

20. Paul Milgram and Fumio Kishino. 1994. A Taxonomy of Mixed Reality Visual Displays. IEICE Transactions on Information Systems 77, 12 (1994), 1321-1329.

21. Jens Müller, Roman Rädle, and Harald Reiterer. 2016. Virtual Objects as Spatial Cues in Collaborative Mixed Reality Environments: How They Shape Communication Behavior and User Task Load. In Proc. CHI 2016. ACM Press, 1245-1249. DOI :

http://dx . doi . org/10.1145/2858036.2858043

22. Jens Müller, Roman Rädle, and Harald Reiterer. 2017. Remote Collaboration with Mixed Reality Displays: How Shared Virtual Landmarks Facilitate Spatial Referencing. In Proc. CHI 2017. ACM Press, 6481-6486. DOI: http://dx.doi.org/10.1145/3025453.3025717

23. Kenton O’hara, Jesper Kjeldskov, and Jeni Paay. 2011. Blended Interaction Spaces for Distributed Team Collaboration. ACM Trans. Comput.-Hum. Interact. 18, 1, Article 3 (May 2011), 28 pages. DOI: http://dx. doi .org/10.1145/1959022 .1959025

24. Phil So. 2017. TLX @ NASA Ames - Home. Website. (2017). Retrieved August 22, 2017 from https://humansystems. arc . nasa.gov/groups/tlx/.

25. Joey Scarr, Andy Cockburn, and Carl Gutwin. 2012. Supporting and Exploiting Spatial Memory in User Interfaces. Foundations and Trends in Human-Computer Interaction 6, 1 (2012), 1-84. DOI: http://dx.doi.org/10.1561/1100000046

26. Dieter Schmalstieg and Tobias Höllerer. 2015. Augmented Reality: Principles and Practice. Addison Wesley Professional, Boston, MA, USA. DOI : http://dx.doi.org/10.1109/VR.2017.7892358

27. Aaron Stafford, Wayne Piekarski, and Bruce Thomas. 2006. Implementation of God-like Interaction Techniques for Supporting Collaboration Between Outdoor AR and Indoor Tabletop Users. In ISMR 2006. IEEE, 165-172. DOI : http://dx.doi .org/10.1109/ISMAR. 2006.297809

28. Aaron Stafford, Bruce H. Thomas, and Wayne Piekarski. 2009. Comparison of Techniques for Mixed-space Collaborative Navigation. In Proc. AUIC 2009. Australian Computer Society, 61-72.

29. Tango Tablet Development Kit User Guide. 2017. (7 April 2017). Retrieved August 22, 2017 from https://developers. google. com/tango/hardware/tablet. 
30. TeamSpeak Communication System. 2017. Welcome to TeamSpeak - TeamSpeak. (2017). Retrieved August 22, 2017 from https://www. teamspeak. com/.

31. Xiangyu Wang and P. S. Dunston. 2011. Comparative Effectiveness of Mixed Reality-Based Virtual Environments in Collaborative Design. IEEE Transactions on Systems, Man, and Cybernetics 41, 3
(2011), 284-296. DOI :

http://dx.doi.org/10.1109/TSMCC. 2010.2093573

32. Xiangyu Wang and Phillip S. Dunston. 2013. Tangible Mixed Reality for Remote Design Review: a Study Understanding User Perception and Acceptance. Visualization in Engineering 8, 1 (2013), 8. DOI: http://dx.doi.org/10.1186/2213-7459-1-8 